\title{
LOS ESTILOS DE APRENDIZAJE: UNA APROXIMACIÓN EN DOCENTES DE EDUCACIÓN BÁSICA EN LAS ESCUELAS DE LA PROVINCIA DE SANTA ELENA
}

\section{LEARNING STYLES: AN APPROACH IN BASIC EDUCATION TEA- CHERS IN THE SCHOOLS OF SANTA ELENA PROVINCE}

\author{
Carlos Cañedo Iglesias, PhD. \\ Prometeo SENESCYT -UPSE \\ ccanedo2013@gmail.com. \\ María Caridad Mederos Machado. MS.c
}

Gina Parrales Loor, MS.c

Docente UPSE

Yuri Ruiz Rabasco, MS.c

Docente UPSE

\section{RESUMEN}

Este trabajo tiene como objetivos conceptualizar el aprendizaje verdaderamente desarrollador y cómo transcurren esos procesos de internalización y externalización, también pretende compartir los resultados de una evaluación utilizando el cuestionario Money - Alonso de estilos de aprendizaje que se realizó con 180 docentes de educación básica de la provincia de Santa Elena. De acuerdo con los resultados obtenidos, se observa, en forma general, que los docentes utilizan un estilo de aprendizaje reflexivo, seguido de los que tienden al estilo activo. Son menos los que evidencian un estilo pragmático y teórico, esta metodología empleada permitió conocer y diagnosticar los estilos de aprendizaje que poseen los profesores que se desempeñan en las escuelas del territorio peninsular y constituye una de las acciones científicas del proyecto de investigación "Estrategias Pedagógicas para lograr una educación inclusiva en las instituciones de Educación Básica en la provincia de Santa Elena, que se desarrolla en la Universidad Estatal Península de Santa Elena Ecuador.

Palabras clave: Estilos de aprendizaje, educación básica, aprendizaje desarrollador.

\section{ABSTRACT}

The objective of this work is to conceptualize learning as a truly developer and how these processes of internalizing and externalizing behave, it also pretends to share the results of an assessment using the Money-Alonso questionnaire of learning styles applied to 180 basic education teachers in the Province of Santa Elena. According to the obtained results, it is generally observed, that teachers use the reflexive learning style, followed by those who use the active learning style. A few of them use a pragmatic and theoretical style, this methodology made it possible to know and diagnose the learning styles of teachers at the schools of the peninsula and becomes one of the scientific actions of this research project "Pedagogical Strategies to achieve an inclusive education in the Basic Education school in the Province of Santa Elena, developed by the Peninsula of Santa Elena State University.

Key words: Learning styles, basic education, developing learning

Recibido: septiembre de 2015 Aprobado: noviembre de 2015

\section{Introducción}

En el mundo actual cobra mayor importancia la educación en las naciones que se proponen alcanzar altos niveles de calidad en la formación de sus ciudadanos. Lograr que cada curso escolar sea cualitativamente superior al anterior, constituye un gran reto para el Estado, el gobierno, directivos y especialmente para los docentes y profesionales de la educación. 
Aunque resultan notables los esfuerzos por alcanzar este empeño, subsisten dificultades, como es el caso del aprendizaje de los estudiantes, para lo cual algunas de las preguntas a realizar pudieran constituir temas de debate en la comunidad científica ecuatoriana:

- ¿Se ha superado realmente el paradigma de la enseñanza tradicional? ¿Por qué?

- ¿Es necesario hacer una reconceptualización del aprendizaje y la enseñanza? ¿Por qué?

- ¿Por qué si tanto se insiste en el aprendizaje, aún existen tantos problemas con el mismo?

- ¿Están los profesores conscientes de la concepción de aprendizaje con la que sus estudiantes aprenden? ¿Conocen la concepción de aprendizaje que sustenta su preparación didáctica? ¿Por qué?

- ¿Están todos los profesores preparados para aplicar estos contenidos de aprendizaje, y por tanto, perfeccionar su accionar didáctico?

Este trabajo tiene como objetivos conceptualizar el aprendizaje verdaderamente desarrollador y como transcurren esos procesos de internalización y externalización y también pretende compartir los resultados de una evaluación de estilos de aprendizaje que se realizó con docentes de educación básica de la Península de Santa Elena.

\section{El aprendizaje}

Existen definiciones derivadas de las diferentes teorías del aprendizaje y tendencias pedagógicas contemporáneas, que dan respuesta a esta pregunta. Desde esta perspectiva de análisis, pueden señalarse aquellas que pertenecen a grandes figuras mundiales que se han dedicado a su estudio, y por tanto son clásicas en el tema, a continuación se exponen algunas de ellas:

"El aprendizaje es una actividad social, y no solo un proceso de realización individual como hasta el momento se había sostenido; una actividad de producción y reproducción del conocimiento mediante la cual el alumno asimila los modos sociales de actividad y de interacción, y más tarde en la escuela, subyacen, los fundamentos del conocimiento científico, bajo condiciones de orientación e interacción social." (1).

Para este autor, el desarrollo de las funciones psíquicas superiores tiene lugar bajo un condicionamiento histórico social, que le imprime características muy particulares en cada individuo, sin descuidar el entorno en que este se desenvuelve. La descripción de las relaciones internas entre el pensamiento y el lenguaje es otro aporte de Vigotsky al desarrollo de las ciencias psicológicas y a las teorías que sustentan el proceso de aprendizaje. Al considerar el papel del lenguaje como modo de representación de la realidad y al mismo tiempo como la forma de concretar y expresar las adquisiciones fundamentales de las personas en el devenir de su pensamiento, se puntualiza en su función de representación y al mismo tiempo de medio de comunicación y emisión de mensajes.

"Aprender es una adquisición permanente de cuerpos estables de conocimientos y de las capacidades necesarias para adquirir tales conocimientos." (2).

Ausubel introduce el concepto de Aprendizaje significativo. Para el autor este proceso está condicionado por varios factores, entre ellos por la significatividad lógica y psicológica del material de aprendizaje y por la disposición del sujeto que aprende.

Al mismo tiempo dichos factores sólo se dan si el nuevo contenido de aprendizaje cobra nuevo significado para el estudiante. Ello quiere decir que para poder contrastar los nuevos contenidos, el sujeto recurre a sus ideas previas sobre el tema en cuestión y trata de contrastar los aprendizajes con ellas.

Según el autor, en la medida en que los conocimientos que se poseen sean más disonantes con los nuevos, resultarán más significativos y entonces habrá mayores posibilidades de que cobren significado y se integren al sistema de conocimientos construidos.

Por ello es importante que los profesores conozcan y profundicen no solo en las ideas previas de los estudiantes, sino en las teorías implícitas de los mismos para poder conducir de manera más eficiente el proceso de construcción de conocimientos.

Las teorías implícitas son sistemas de ideas previas elaboradas por los sujetos que aprenden, de modo que las mismas están en la base de la construcción de los conocimientos.

"Las personas construyen representaciones de la realidad y utilizan sus procesos cognitivos para interpretar situaciones, predecir y comprender el comportamiento de otras personas y planificar el propio. Esto supone no sólo aceptar que la mente es un sistema de procesamiento de información que adquiere significado dentro de una interac- 
ción social, o si se prefiere, en presencia de otras mentes. No es una propiedad que se puede aplicar a una materia, sino que sólo se puede aplicar a una materia organizada socialmente". (3)

El aprendizaje supone continuas reconstrucciones mentales de lo ya conocido, pero siempre se reedifica el conocimiento sobre la base de la teoría implícita. Es un proceso inconsciente del individuo, pero al mismo tiempo es un proceso que incita a la acción, una vez que se convierte en actitud.

"El aprendizaje se caracteriza por la adquisición de los mediadores o amplificadores culturales, creados, almacenados y transmitidos por una cultura específica y que le permite al hombre trascender su experiencia individual. El aprendizaje determina el desarrollo como incremento de la capacidad del individuo para manejar información del medio ambiente." (4).

Por este motivo es que el enfoque constructivista no puede aislarse del entorno de aprendizaje, entendido este no solo como los escenarios de la propia escuela, sino que los mismos se amplían hacia otras esferas, incluso algunas intangibles como suele ser el cyber espacio.

Las condiciones del entorno de aprendizaje va a estar determinando la calidad del proceso, de modo que las mismas pueden acelerar o demorar la construcción de conocimientos. Es imprescindible que para aprender en términos formales, la institución educativa garantice un mínimo de condiciones y contribuir así a que la reacción de aprender se desarrolle de manera pertinente ante la actividad de enseñar que debe facilitar la institución educativa.

"Aprender es el proceso por el cual se origina o cambia una actividad, mediante la reacción a una situación dada, siempre que las características del cambio en curso no puedan ser explicadas con apoyo en tendencias, reacciones innatas, en la maduración por cambios corporales del organismo, por ejemplo, la fatiga, las drogas, etc." (5).

Aunque aún se encuentra en proceso de conformación la proyección ideográfica de la Pedagogía latinoamericana, en la actualidad existen conceptualizaciones acerca del aprendizaje que se alinean con las exigencias del mundo moderno, estimulando su sentido activo y crítico. "Enseñar no es transferir conocimiento, sino crear las posibilidades para su producción o su construcción". (6)

A partir de esta premisa es de considerar que como proceso humano, el aprendizaje transcurre en un nivel de complejidad donde las representaciones de la realidad aprendida están muy relacionadas con las experiencias previas, los conocimientos y las necesarias contradicciones propias de cada individuo.

"En un sentido amplio, el aprendizaje se entiende como un proceso dialéctico en el que, como resultado de la práctica, se producen cambios relativamente duraderos y generalizables, y a través del cual el individuo se apropia de los conocimientos y las formas de pensar, sentir y actuar construidas en la experiencia en su contexto histórico cultural con el fin de adaptarse a la realidad y/o transformarla." (7).

La concepción de este autor pone de manifiesto algunas ideas que identifican el aprendizaje en todas sus etapas y no solo en su resultado, ellos son:

1- Carácter dialéctico del proceso, no es lineal, es en una espiral continua.

2- Estabilidad de lo que se aprende.

3- Articulación de lo aprendido con la experiencia individual y con el entorno socio cultural.

4- Lo aprendido como elemento de transformación.

La realidad nos indica que conceptualizar al aprendizaje desde las diversas concepciones existentes, ha traído como consecuencias un sinnúmero de dificultades para su comprensión:

El aprendizaje ha sido comprendido indistintamente como un proceso que:

a) Se encuentra restringido al espacio de la institución escolar (aprendizaje formal), a determinadas etapas exclusivas de la vida (a las que preparan para la vida profesional, adulta);

b) Que maximiza lo cognitivo, lo intelectual, lo informativo, los saberes, sobre lo afectivo emocional, lo ético y lo vivencial, y el saber hacer;

c) Que se realiza individualmente, aunque, paradójicamente, no se tenga en cuenta o se subvalore al individuo;

d) Como una vía exclusiva de socialización, más que de individualización, de personalización, de construcción y descubrimiento de la subjetividad;

e) Como adquisición de conocimientos, hábitos, destrezas y actitudes para adaptarse al medio, 
más que para aprender a desarrollarse, a aprender y a crecer."

Desde esta perspectiva de análisis (7) ofrece algunas pautas a seguir en la comprensión del aprendizaje.

- Aprender es un proceso que ocurre a lo largo de toda la vida, y que se extiende en múltiples espacios, tiempos y formas. El aprender está estrechamente ligado con el crecer de manera natural. El proceso de aprendizaje es tanto una experiencia intelectual como emocional. Engloba la personalidad como un todo visto desde el paradigma de la complejidad. Se construyen en él los conocimientos, destrezas, capacidades, se desarrolla la inteligencia, pero de manera inseparable, es una fuente de enriquecimiento afectivo, donde se forman sentimientos, valores, convicciones, ideales, donde emerge la propia persona y sus orientaciones ante la vida.

- Aunque el punto central y el principal instrumento del aprender es el propio sujeto que aprende, aprender es un proceso de participación, de colaboración y de interacción. En el grupo, en la comunicación con los otros, las personas desarrollan el compromiso y la responsabilidad, individual y social, elevan su capacidad para reflexionar divergente y creadoramente, para la evaluación crítica y autocrítica, para solucionar problemas y tomar decisiones. El papel protagónico y activo de la persona no niega, en resumen, la mediación social.

- En el aprendizaje cristaliza continuamente la dialéctica entre lo histórico-social y lo individual-personal; es siempre un proceso activo de re-construcción de conocimientos y de descubrimiento del sentido personal y de la significación vital que tienen los mismos para los sujetos.

- Aprender supone el tránsito de lo externo a lo interno -en palabras de Vigotsky, de lo interpsicológico a lo intrapsicológico de la dependencia del sujeto a la independencia, de la regulación externa a la autorregulación. Tipos de aprendizaje.

De la misma forma que ocurre con la definición de aprendizaje, sucede con los tipos de aprendizajes, es decir, existen diversas clasificaciones al respecto. Cañedo (7) señala que muchas veces los diversos tipos de aprendizaje que se definen no tienen explícito el criterio en que se sustentan, y los tipifica de la siguiente forma:
Tipo 1_ Atendiendo al proceso Psíquico que se emplee se clasifica en Aprendizaje Cognitivo y Aprendizaje Memorístico.

Tipo 2_Atendiendo al carácter consciente o no consciente se clasifica en Aprendizaje Reflexivo y Aprendizaje Incidental.

Tipo 3_Atendiendo a la medida en que se integra a intereses y vida personal del sujeto se clasifica en Aprendizaje Significativo.

Tipo 4_ De acuerdo al procedimiento para aprender se clasifica en Aprendizaje Clásico o Instrumental, Aprendizaje por Ensayo- Error, Aprendizaje por descubrimiento.

Tipo 5_ De acuerdo al objeto hacia el que se dirige se clasifica en Aprendizaje de serie, Aprendizaje de Conceptos y Aprendizaje de solución de problemas.

Tipo 6_ Por las condiciones en que se produce se clasifica en Aprendizaje grupal y Aprendizaje individual.

Es necesario indicar que (8) - pionero de las investigaciones sobre Inteligencias Múltiples-, aunque presenta y afirma la necesidad secuencial de ocho tipos de aprendizaje, concede mayor importancia al aprendizaje de conceptos, principios y solución de problemas, por ser los aprendizajes característicos de la instrucción escolar y constituir el eje del comportamiento inteligente de la persona, según su opinión.

Además de los tipos de aprendizaje se conceptualizan los estilos.

\section{Estilos de aprendizaje}

Según Keefe en 1988, citado(9), "los estilos de aprendizaje son definidos como los rasgos cognitivos, afectivos y fisiológicos que sirven como indicadores relativamente estables, de cómo los alumnos perciben, interaccionan y responden a sus ambientes de aprendizaje". Este concepto ratifica la idea de todos los procesos psíquicos de la persona que participan en el proceso y que le otorgan la categoría de ser absolutamente individual y de una fuerte carga subjetiva.

Por su parte (9), clasifican los estilos de aprendizaje en Activo, Reflexivo, Teórico y Pragmático, y consideran que cada uno de ellos representa una preferencia específica en el modo de aprender.

Se refieren al estilo Activo cuando se evidencia implicación activa y sin prejuicios en nuevas ex- 
periencias de aprendizaje. Consideran el Reflexivo cuando existe observación de las experiencias desde diversas perspectivas, en este caso hay prioridad de la reflexión sobre la acción. El estilo Teórico supone un enfoque lógico de los problemas y la integración de la experiencia dentro de teorías complejas. El estilo Pragmático está relacionado con la experimentación y aplicación de ideas.

Aunque estos autores refieren los estilos mencionados plantean la idea de que las personas no tienen un solo estilo, sino la combinación de ellos, por lo que se habla de Perfil de Aprendizaje, dadas las características psicológicas que integran el estilo.

Bajo estos criterios desarrollaron un cuestionario, conocido como CHAEA, que permite acercarse al Perfil de cada persona. Este cuestionario ha sido diseñado para identificar el estilo preferido de aprendizaje. El mismo resulta un valioso instrumento de evaluación del proceso y ha sido utilizado y comprobada su fiabilidad en diferentes sistemas educativos y permite feed back para mejorar la calidad de la educación.

\section{Análisis de resultados}

En el estudio se aplicó el cuestionario Money Alonso de Estilos de Aprendizaje, adaptado por la profesora Ángela Rosa Hernández, a 100 profesores de Educación Básica de la Provincia Santa Elena.

Los datos obtenidos en el estudio fueron capturados a partir de una hoja de cálculo del programa Excel, del paquete ofimático de Microsoft Office.

Mediante los resultados obtenidos se pudo apreciar que, en sentido general, predominan los docentes con un estilo de aprendizaje reflexivo, seguido de los que tienden al estilo activo y pragmático. Al final, se evidencia con menor valor el estilo teórico. Ver tabla No.1.

Tabla No.1. Promedios de preferencias en las respuestas que significan Estilos de Aprendizaje.

\begin{tabular}{|c|c|c|c|c|c|}
\hline Preferencia & $\begin{array}{l}\text { Muy } \\
\text { baja }\end{array}$ & Baja & Moderada & Alta & $\begin{array}{l}\text { Muy } \\
\text { alta }\end{array}$ \\
\hline Activo & $0-6$ & $7-8$ & $9-12 / 9.75$ & 13-14 & $15-20$ \\
\hline Reflexivo & $0-10$ & $\begin{array}{c}11-13 / \\
11.2\end{array}$ & $14-17$ & $18-19$ & 20 \\
\hline Teórico & $\begin{array}{l}0-6 / \\
5.65\end{array}$ & $7-9$ & $10-13$ & 14-15 & $16-20$ \\
\hline Pragmático & $0-8$ & $\begin{array}{l}9-10 / \\
9.25\end{array}$ & $11-13$ & $14-15$ & $16-20$ \\
\hline
\end{tabular}

Elaborada por: Equipo de investigación.

Sólo se puede hablar de tendencias, dado que este cuestionario fue aplicado por vez única y bajo condiciones especiales, en que los sujetos de la investigación se encontraban en posición de estudiantes, pues este es también el resultado de jornadas de capacitación a las que fueron convocados por la Universidad Estatal Península de Santa Elena.

Estas circunstancias y los conocimientos que se abordaron en las jornadas de capacitación, pueden haber modificado estos resultados, aunque es de considerar que los estilos de aprendizaje no son únicos e inamovibles, sino que los mismos se transforman en dependencia de las condicionantes psicológicas de cada persona y los escenarios del aprendizaje, entre otras razones.

Esto último explica por qué los individuos que han recibido iguales tratamientos pedagógicos expresan estilos diferentes al aprender.

Aunque el instrumento utilizado fue creado para la evaluación del estilo de aprendizaje individual, con fines de tener una visión general de lo que ocurre en los estilos de aprendizaje de este grupo, se realizó un análisis de las frecuencias de los ítems más señalados positivamente por parte de los mismos.

Al analizar las sumatorias de cada uno de los 80 ítems del instrumento aplicado, se infiere que los estilos predominantes como grupo son Reflexivo y Activo. Son los ítems que obtuvieron más puntuaciones en sentido general, aunque hay una variedad notable de estilos dentro de este gran grupo. Ver gráfico No.1

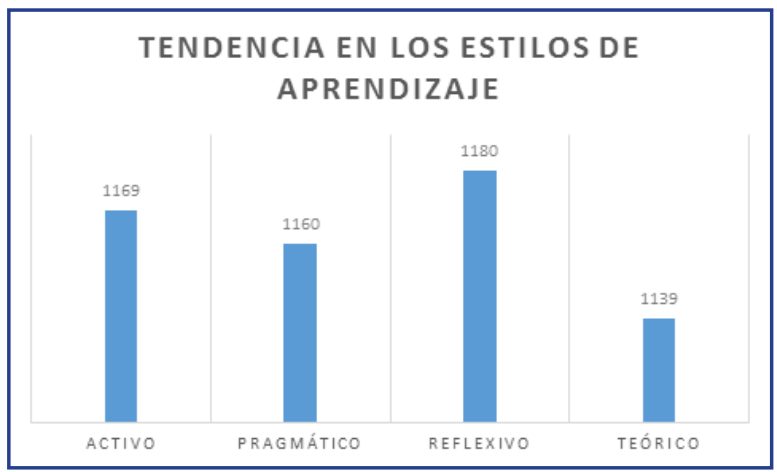

Gráfico No. 1. Tendencia en los estilos de aprendizaje del grupo estudiado.

Elaborada por: Equipo de investigación.

No se asegura que en todos los sujetos estudiados predominan dichos estilos, pero queda claro, que en ese escenario de aprendizaje, la tendencia de estilo es la mencionada. Dicha propensión de hecho puede ser modificada con el paso de los días y cambie la dinámica de conocimientos a los que fueron acercados los docentes en esas sesio- 
nes de capacitación al volver a su rutina cotidiana.

Lo cierto es que se constituye en estilo, cuando las acciones son frecuentes y llegan a modelarse como las maneras de aprender de cada sujeto para diferentes circunstancias.

Como se puede apreciar en el mismo gráfico los estilos Pragmático y Teórico no constituyen tendencia en este grupo, aun cuando durante las sesiones de trabajo se les indicaba el estudio y se inducía la construcción de conocimientos sobre estas temáticas. El estilo pragmático sin embargo es de una puntuación muy cercana a la del Práctico, lo que se explica a partir del sentido personal que cobra ser Activo y ser Pragmático, aun cuando los sujetos no sabían cuáles serían las categorías diagnósticas al responder las preguntas del test.

El estilo Teórico, que, sin embargo, debía aproximarse más al Reflexivo, por lo que de estudio y conocimiento implica la acción de reflexionar, aparece como el menos señalado a partir de las respuestas ofrecidas.

Al tratarse de docentes, aunque en este caso en situación de estudiantes y sujetos que aprenden, se esperaba por parte del equipo de investigación que existiera una puntuación mayor para este estilo Teórico, pues su sistema de conocimientos debe estar más próximo a las habilidades de aprender también desde posiciones que impliquen reconocimiento y ejercicio de la teoría de aprender.

Como conclusión, es importante considerar que existen múltiples conceptualizaciones de los tipos de aprendizaje y clasificaciones de tipos y estilos. Todos coinciden en su carácter dialéctico y en las posibilidades que su estudio ofrece al mejoramiento de la calidad educativa.

La idea final es que los estilos pueden ser desarrollados en cada persona a lo largo de la vida y al mismo tiempo son susceptibles de ser enseñados y mejorados, en la medida en que estos se concientizan por los sujetos que aprenden.

\section{Referencias Bibliográficas}

(1) Vigotsky, L. S. Pensamiento y lenguaje, Madrid. Editorial Paidós. (1978)

(2) Ausubel, D. Teoría del Aprendizaje significativo. Paidós. España. (1991).

(3) Rodrigo, M.J. Representaciones y procesos en las teorías implícitas. En: M.J. Rodrigo, A. Ro- dríguez y J. Marrero, Las teorías implícitas. Una aproximación al conocimiento cotidiano (pp.95122) Madrid: Visor. (1993).

(4) Bruner, J. Actos de significado. Más allá de la Revolución Cognitiva. Alianza. (2006).

(5) Hilgard, E.: Teorías del aprendizaje. Edición Revolucionaria. Inst. Cubano del Libro, C. de La Habana, (1961).

(6) Freire, P. Pedagogía de la autonomía. Paz e Terra SA Ciudad: Sao Paulo.(2009).

(7) Cañedo, C. M. El paso del Sistema Real al Esquema de Análisis. Una estrategia Didáctica en la formación del ingeniero Mecánico (Tesis en opción del título de Doctor en Ciencias Pedagógicas, Cfgos,.--120p).(2004).

(8) Gagné, R.M. Diseño de la enseñanza para un aprendizaje eficaz. McGraw-Hill Interamericana, México, (1993).

(9) Alonso, C. M., Gallego, D.J. y Honey, P. Los estilos de aprendizaje. Procedimientos de diagnóstico y mejora. España: Ediciones Mensajero. (1997). 